\title{
Correlation of anthropometric indices related to obesity with pulmonary function tests in female medical students
}

\author{
Harleen Kaur ${ }^{1}$, Archana Goel ${ }^{2, *}$, Nidhi Puri ${ }^{3}$ \\ ${ }^{1}$ Student, Maharishi Markandeshwar Institute of Medical Sciences and Research, Ambala, Haryana, ${ }^{2}$ Associate Professor, Dept. \\ of Anatomy, Adesh Medical College and Hospital, Mohri, Kurukshetra, Haryana ${ }^{3}$ Professor, Dept. of Anatomy, Dr. Yashwant \\ Singh Parmar Govt. Medical college, Nahan
}

*Corresponding Author:

Email: archanagoel123@yahoo.in

Received: $14^{\text {th }}$ December, 2017

Accepted: $16^{\text {th }}$ January, 2018

\begin{abstract}
Obesity is a major contributor to many classes of disease including pulmonary diseases. The purpose of the study to predict the risk of future pulmonary diseases by using indices related to obesity. Various such indices have been used in previous studies but their relative importance remains unknown. The present study utilized four different anthropometric parameters and calculated their correlation with the pulmonary function tests. This study was also carried out to examine the relative importance of these parameters in prediction of pulmonary functions in a group of female students. This cross-sectional study included 200 female medical students. Anthropometric indices used in this study included: Body Mass Index (BMI), Waist to Hip Ratio (WHR), Waist to Height Ratio (WHtR) and Waist Circumference (WC). Pulmonary function tests were performed using a computerized spirometer. Body Mass Index and Waist to Height Ratio showed a significant negative correlation with Forced Expiratory Flow and ratio of Forced Expiratory Volume in one second: Forced Vital Capacity(FEV 1 : FVC). While Waist Circumference showed a significant negative correlation only with ratio of Forced Expiratory Volume in one second: Forced Vital Capacity. Hence, Body Mass Index, Waist to Height Ratio and Waist circumference can be regarded as most reliable indicators of obesity which leads to decrease in pulmonary functions.
\end{abstract}

Keywords: Body mass index, obesity, pulmonary function tests, Waist Circumference, Waist Hip Ratio, Waist Height Ratio.

\section{Introduction}

Pulmonary diseases are one of the most common and much visited complaints to any general physician. In India, coronary pulmonary disease only has a prevalence rate ranging from 2 to $22 \%$ in males and 1.2 to $19 \%$ in females. ${ }^{1}$ For monitoring the pulmonary functions, spirometry is the most common test performed on a daily basis.

Also obesity, which was earlier considered a disease of affluent society, has now reached epidemic levels globally. ${ }^{2}$ This obesity is a major contributor to different classes of disease, including pulmonary diseases.

Anthropometric indices related to obesity such as body mass index (BMI), waist circumference (WC), waist hip ratio (WHR) and waist height ratio (WHtR) can help in predicting future risk of developing pulmonary disease. Hence, these parameters were used in this study to predict the future pulmonary disease risks among female medical students of north India. Any such collaborated study using variety of anthropometric indices as indicators of obesity and assessing its relation with pulmonary function tests was not found in this area of north India.

\section{Materials and Methods}

A cross-sectional study was conducted among 200 female medical students. There were 325 female medical students in medical college at the time of study. Of these female medical students, 39 students were excluded on applying exclusion criteria i.e. Subjects suffering from any respiratory disease, smokers, wheel chair bound or disabled subjects and subjects with history of drug intake were excluded from the study.

Prior informed consent was obtained from all the students in the study sample in writing, both in English and vernacular. 86 students however did not want to be part of the study and remaining 200 students thus formed our study sample. Three readings of each of the measurement were taken and then their average value was calculated to ensure the accuracy. A weighing scale of sensitivity and specificity was used for measuring weight. Other anthropometric measurements were done using a flexible metallic measuring tape. Computerized spirometer, Spiro-Excel (Medicaid Systems Chandigarh) was used for recording the pulmonary function tests.

Following measurements were taken:

1. Height (m): Measurement of maximum distance from floor to the highest point on head, when the subjects were facing directly ahead and with shoes removed feet together and arms by sides. Heels, buttocks and upper back were in contact with wall when measurement was made.

2. Weight (kg): Subjects stood on calibrated weighing scales with minimum body movements and hands by their sides. Shoes and excess clothing was removed. 
3. Waist Circumference $(\mathrm{cm})$ : taken at the level mid way between the lower rib margin and iliac crest with the tape all around the body in horizontal position.

Normal: $\leq 80 \mathrm{~cm}$

High risk: $>80 \mathrm{~cm}^{3}$

4. Hip Circumference $(\mathrm{cm})$ : taken over minimal clothing, at the level of greatest protrusion of gluteal (buttocks) muscles. The subjects stood erect with weight evenly distributed on both the feet with legs slightly parted.

For spirometry, the subjects were informed about the whole maneuver and were encouraged to practice this maneuver before doing the pulmonary test. The test was performed with the subjects in sitting position by using a nose clip. This procedure was repeated and the best of three readings was considered for analysis. The parameters recorded were Peak Expiratory Flow Rate (PEFR), Forced Expiratory Flow $\left\{\mathrm{FEF}_{(25-75 \%)}\right\}$, Forced Expiratory Volume in One Second $\left(\mathrm{FEV}_{1}\right)$, Forced Vital Capacity (FVC), $\mathrm{FEV}_{1}$ : FVC. Respiratory Rate (RR) was also noted. At least three tests of acceptable effort were performed to ensure reproducibility. The best trial was selected from three reproducible trials for reporting.

After taking all parameters following indices were calculated:

1. Body mass index $(\mathrm{BMI})^{4,5}$ : Weight $(\mathrm{Kg}) /$ Height $\left(\mathrm{m}^{2}\right)$

Normal: $18.50-22.99 \mathrm{~kg} / \mathrm{m}^{2}$

Over weight: $23.00-24.99 \mathrm{~kg} / \mathrm{m}^{2}$

Obesity: $\geq 25.00 \mathrm{~kg} / \mathrm{m}^{2}$

2. Waist Hip Ratio $(\text { WHR })^{5}$ : Waist circumference $(\mathrm{cm})$ /Hip circumference $(\mathrm{cm})$

Normal: $<0.81$

High WHR: $\geq 0.81$

3. Waist-Height Ratio (WHtR) ${ }^{6}$ : Waist circumference $(\mathrm{cm}) /$ Height $(\mathrm{cm})$

Normal: $<0.5$

High: $\geq 0.5$

\section{Data Analysis}

Appropriate data entry and statistical analysis was performed on SPSS (statistical package for social sciences) software version 23. Data was summarized using descriptive statistics. Continuous variables were presented as mean and S.D. while categorized variable as number and percentage. Correlation (pearsons 2 tailed correlation) between anthropometric parameters and obesity related indices was found. $\mathrm{P}$ value of $<0.05$ was taken as statistically significant. The approval of ethical committee of the institution was also taken.

\section{Results and Discussion}

The study sample included 200 female medical students. The mean and standard deviation values of various anthropometric measures and pulmonary function tests are as given in table 1.
The study population was then divided into different categories on the basis of their anthropometric measures. Number of candidates belonging to each category along with the mean and standard deviation of pulmonary functions in each category is given in table 2 . Table 2 also shows the prevalence of deranged anthropometric indices in study population.

Also the prevalence of normal deranged $(<0.7)$ values of $\mathrm{FEV}_{1}: \mathrm{FVC}^{7}$ is given in table 3 .

Correlation of pulmonary function tests with various anthropometric parameters was calculated and is given in table 4. Also, significance of difference in various groups was calculated using Student's t-test and is given in table 5.

BMI values showed significant negative correlation with values of FEF and FEV: FVC (as shown in table 4) i.e. as BMI increased, values of FEF and FEV: FVC decreased. There was a significant difference between PEFR, FEF and FEV: FVC values across the three groups of BMI (table 5). Extremely significant difference $(p \leq 0.0001)$ was observed for FEV: FVC among overweight and obese candidates (table 5). The values of FVC and FEV showed no significant differences among three categories of BMI. WHR showed no significant correlation with any of the parameters of pulmonary function tests (table 4). None of the values of pulmonary function tests showed significant difference between normal and high WHR (table 5). WHtR showed significant negative correlation with FEF and FEV: FVC (table 4) which means that as WHtR increased, the values of FEF and FEV: FVC decreased. The values of FEV: FVC were significantly less in candidates with higher WHtR (table 5). The values of FEV: FVC were in significant negative correlation with the values of WC (table 4). Otherwise, the values of pulmonary function tests showed no significant difference between two categories of WC (table 5).

Obesity is a major health hazard across the world. It affects many areas of clinical complications such as diabetes, vascular diseases, osteoarthritis, etc. But in this context, less emphasis has been given on the effect of obesity on respiratory system. ${ }^{8}$ In this study an attempt was made to find out whether there is an increased risk of respiratory problems in overweight and obese individuals.

Pulmonary function measurements are frequently related to body size and age, where height is a proxy for chest size, and age reflects maturity. ${ }^{9}$ It is due to this reason that every individual has different range of normal values. In contrast, $\mathrm{FEV}_{1}: \mathrm{FVC}$ has been assigned a cut- off value of 0.70 and the prevalence of normal and deranged $\mathrm{FEV}_{1}: \mathrm{FVC}$ in this study population is provided in table 5 .

This study was based on the hypothesis that any increase in the anthropometric parameters related to obesity (BMI, WHR, WHtR and WC) will lead to a decrease in pulmonary functions which may be due to 
number of effects that obesity has on pulmonary functions. The presence of fatty tissue around rib cage, abdomen and in perivisceral space loads the chest wall, thus reducing pulmonary functions. ${ }^{8}$ Our study revealed significant decrease in pulmonary function tests in overweight and obese female students who do not have any known obstructive airway disease and thus supported the hypothesis.

In this study, BMI showed a significant negative correlation with FEF and $\mathrm{FEV}_{1}$ : FVC (table 2). FEF is an indicator of mid expiratory flow rate and it was taken as a marker of obstructive lung diseases like asthma and COPD. Therefore, it can be said that obesity has significant impact on respiratory problems.

Other parameters like WHR, WHtR and WC were used to assess abdominal obesity. The values of PEFR, FEF, FEV ${ }_{1}$ and $\mathrm{FEV}_{1}$ : FVC were observed to be less in individuals with higher WHR although no significant correlation was found (table 4). In contrast, WHtR showed significant negative correlation with FEF and $\mathrm{FEV}_{1}$ : FVC while WC showed significant negative correlation with $\mathrm{FEV}_{1}$ : FVC of the concerned female students (table 4). This supports the hypothesis that an excess of abdominal fat may limit the movement of diaphragm and hence lead to a decrease in pulmonary function.

It is but astonishing that a significant correlation could be found in this population as pulmonary function test is largely based on voluntary effort. It can be assumed that the real effect will be even greater in magnitude. Better methods to assess pulmonary functions should be sought for. Also, this study indicated BMI and WHtR to be better and more reliable indicators of pulmonary health than the other indices.
The findings in this study suggest that there is significant impairment of pulmonary functions in overweight and obese population due to limited expansion of thoracic cavity which leads to possibility of small airway diseases. But these effects of obesity might be reversible and weight loss could possibly improve lung functions. Further studies showing the effect of weight loss on improvement of pulmonary functions are need of the hour. More researches with greater sample size and advanced methods to measure pulmonary function test values can help in assessing the correlation in a better manner. Assessing the effect of weight loss can also help in creating awareness about obesity related health issues.

Table 1: Mean and Standard Deviation $($ Mean \pm SD) of various anthropometric parameters and indices related to obesity, various pulmonary function test values and respiratory rate

\begin{tabular}{|l|c|}
\hline \multicolumn{1}{|c|}{ Data } & Mean \pm SD \\
\hline Height $(\mathrm{m})$ & $1.59 \pm 0.06$ \\
\hline Weight $(\mathrm{kg})$ & $57.98 \pm 11.66$ \\
\hline Waist circumference $(\mathrm{cm})$ & $77.1 \pm 10.94$ \\
\hline Hip circumference $(\mathrm{cm})$ & $96.29 \pm 9.72$ \\
\hline BMI $\left(\mathrm{kg} / \mathrm{m}^{2)}\right.$ & $22.83 \pm 4.48$ \\
\hline WHR $(\mathrm{cm} / \mathrm{cm})$ & $0.8 \pm 0.06$ \\
\hline WHtR $(\mathrm{cm} / \mathrm{cm})$ & $0.48 \pm 0.07$ \\
\hline PEFR & $5.94 \pm 1.31$ \\
\hline FEF & $4.26 \pm 0.96$ \\
\hline FVC & $2.67 \pm 0.52$ \\
\hline FEV & $2.43 \pm 0.49$ \\
\hline FEV $_{1}:$ FVC & $0.91 \pm 0.07$ \\
\hline Respiratory rate (per minute) $^{2}$ & $14.86 \pm 1.71$ \\
\hline
\end{tabular}

Table 2: Mean and SD of PFT values in different categories of anthropometric measures

\begin{tabular}{|c|c|c|c|c|c|c|c|}
\hline & $\mathrm{N}$ & Prevalence & PEFR & FEF & FVC & $\mathrm{FEV}_{1}$ & FEV:FVC \\
\hline \multicolumn{8}{|l|}{ BMI } \\
\hline Normal & 113 & $56.5 \%$ & $6.15 \pm 1.09$ & $4.45 \pm 0.8$ & $2.66 \pm 0.48$ & $2.47 \pm 0.43$ & $0.93 \pm 0.04$ \\
\hline Overweight & 35 & $17.5 \%$ & $5.50 \pm 1.59$ & $4.03 \pm 1.05$ & $2.62 \pm 0.61$ & $2.31 \pm 0.60$ & $0.88 \pm 0.12$ \\
\hline Obese & 52 & $26 \%$ & $5.77 \pm 1.45$ & $3.98 \pm 0.99$ & $2.72 \pm 0.52$ & $2.44 \pm 0.50$ & $0.89 \pm 0.07$ \\
\hline \multicolumn{8}{|l|}{ WHR } \\
\hline Normal & 116 & $58 \%$ & $5.97 \pm 1.33$ & $4.35 \pm 0.95$ & $2.66 \pm 0.55$ & $2.44 \pm 0.51$ & $0.92 \pm 0.07$ \\
\hline High & 84 & $42 \%$ & $5.90 \pm 1.28$ & $4.12 \pm 0.95$ & $2.69 \pm 0.46$ & $2.43 \pm 0.45$ & $0.90 \pm 0.08$ \\
\hline \multicolumn{8}{|l|}{ WHtR } \\
\hline Normal & 130 & $65 \%$ & $6.01 \pm 1.17$ & $4.34 \pm 0.88$ & $2.67 \pm 0.51$ & $2.45 \pm 0.45$ & $0.92 \pm 0.05$ \\
\hline High & 70 & $35 \%$ & $5.79 \pm 1.51$ & $4.08 \pm 1.05$ & $2.67 \pm 0.52$ & $2.38 \pm 0.53$ & $0.89 \pm 0.10$ \\
\hline \multicolumn{8}{|l|}{ WC } \\
\hline Normal & 137 & $68.5 \%$ & $5.93 \pm 1.32$ & $4.31 \pm 0.96$ & $2.63 \pm 0.53$ & $2.41 \pm 0.50$ & $0.92 \pm 0.08$ \\
\hline High & 63 & $31.5 \%$ & $5.95 \pm 1.29$ & $4.14 \pm 0.94$ & $2.76 \pm 0.48$ & $2.48 \pm 0.46$ & $0.90 \pm 0.08$ \\
\hline
\end{tabular}

$\mathrm{n}$ is the number of candidates.

Table 3: Prevalence of deranged FEV 1 : FVC

\begin{tabular}{|l|c|c|}
\hline \multicolumn{1}{|c|}{ Category } & n & Prevalence \\
\hline Normal FEV $_{1}:$ FVC & 195 & $97.5 \%$ \\
\hline Deranged FEV $_{1}:$ FVC & 5 & $2.5 \%$ \\
\hline
\end{tabular}

$\mathrm{n}$ is the number of candidates. 
Table 4: Correlation of the anthropometric indices related to obesity with various pulmonary function tests

\begin{tabular}{|l|c|c|c|c|c|}
\hline & PEFR & FEF & FVC & FEV $_{1}$ & FEV:FVC \\
\hline BMI & -0.076 & $-0.146^{*}$ & 0.121 & 0.031 & $-0.243^{* *}$ \\
\hline WHR & -0.006 & -0.057 & 0.024 & 0.018 & -0.035 \\
\hline WHtR & -0.076 & $-0.147^{*}$ & 0.036 & -0.023 & $-0.167^{*}$ \\
\hline WC & -0.027 & -0.106 & 0.123 & 0.061 & $-0.165^{*}$ \\
\hline
\end{tabular}

$\{* *$. Correlation is significant at the 0.01 level (2-tailed); *. Correlation is significant at the 0.05 level (2-tailed) $\}$.

Table 5: Comparison of PFT values in normal and deranged categories of various anthropometric indices

\begin{tabular}{|l|c|c|c|c|c|}
\hline \multicolumn{1}{|c|}{ P values of........... } & PEFR & FEF & FVC & FEV $_{1}$ & FEV:FVC \\
\hline $\begin{array}{l}\text { BMI (NORMAL AND } \\
\text { OVERWT) }\end{array}$ & $0.0068^{* *}$ & $0.0131^{*}$ & 0.6153 & 0.0838 & $0.0003^{* * *}$ \\
\hline $\begin{array}{l}\text { BMI (NORMAL AND } \\
\text { OBESE) }\end{array}$ & 0.0628 & $0.0014^{* *}$ & 0.5458 & 0.6933 & $0.0001^{* * *}$ \\
\hline $\begin{array}{l}\text { WHR (NORMAL AND } \\
\text { HIGH) }\end{array}$ & 0.7094 & 0.0926 & 0.6843 & 0.8859 & 0.1573 \\
\hline WHtR (NORMAL AND & & & & & \\
HIGH) & 0.2803 & 0.066 & 1 & 0.3358 & $0.0014^{* *}$ \\
\hline WC (NORMAL AND HIGH) & 0.9203 & 0.2431 & 0.0988 & 0.347 & 0.0777 \\
\hline
\end{tabular}

*-statistically significant; $* *$-very significant; $* * *$-extremely significant.

\section{Conclusion}

Our study found that pulmonary functions like PEFR, FEF and $\mathrm{FEV}_{1}$ : FVC were significantly affected by anthropometric parameters related to obesity in female medical students. BMI, WHtR, WC had a significant negative correlation with $\mathrm{FEF}$ and $\mathrm{FEV}_{1}$ : FVC. No negative correlation of WHR with pulmonary function tests was observed. It can be stated that abdominal obesity leads to a decrease in pulmonary functions by decreasing the compliance of lungs and diaphragm.

The correlation found in this study, which are much easier to measure will in itself be an indicator of pulmonary health. The health care in remote areas will benefit a lot, as there are minimum of facilities available.

\section{Limitations of the Study}

Due to small sample size the results obtained in this study may not be the representative data for whole population. But our primary aim is to generate preliminary data and this will provide useful information and will be helpful in further study.

\section{Conflicts of Interest}

Authors declare no conflict of interests.

\section{Acknowledgement}

This research has been approved by Indian Council of Medical Research (ICMR). The project received funding from ICMR under the scheme of Short Term Studentship (STS). chronic obstructive pulmonary disease and its association with smoking." Indian J Chest Dis Allied Sci. 2001;43:13947.

2. World Health Organisation (WHO). Obesity and Overweight. Geneva: WHO; 2003.

3. Lean ME, Han TS, Morrison CE. "Waist circumference as a measure for indicating need for weight management." BMJ. 1995;311(6998):158-61.

4. Patil SP, Sukumaran S, Bhate A, Mukherji A, Chandrakar S. "Correlation of blood sugar with waist circumference and body mass index in an Indian population." Global J Pharmacol. 2012;6(1):08-11.

5. Snehalata C, Viswanathan V, Ramachandran A. "Cutoff values for normal anthropometric variables in Asian Indian Adults." Diabetes Care. 2003;26(5):1380-4.

6. Deshmukh PR, Gupta SS, Dongre AR, Bharambe MS, Maliye C, Kaur S, et al. 2006. "Relationship of anthropometric indicators with blood pressure levels in rural Wardha." Indian J Med Res. 2006;123:657-64.

7. Global Strategy for the Diagnosis, Management and Prevention of COPD, Global Initiative for Chronic Obstruction Lung Disease (GOLD). 2007.

8. Salome CM, King GG, Bernard N. "Physiology of obesity and effects on lung function.” J Appl Physiol. 2010;108(1):206-11.

9. Stanojevic S, Wade A, Stocks J. "Reference values for lung function: past, present and future." European Resp J. 2010;36:12-19.

\section{References}

1. Jindal SK, Aggarwal AN, Gupta D. "A review of the population studies from India to estimate national burden of 